\title{
GESTIÓN DE DATOS DE INVESTIGACIÓN EN LOS OBSERVATORIOS DE TURISMO REGIONAL EN CHILE ${ }^{1}$
}

\author{
Patricio Padilla* \\ Universidad de La Frontera. Chile \\ https://orcid.org/0000-0002-4651-3253 \\ Sergio Sánchez* \\ Universidad de La Frontera. Chile \\ https://orcid.org/0000-0002-0154-1496 \\ Jorge Hernández* \\ Universidad de La Frontera. Chile \\ https://orcid.org/0000-0002-0921-7324 \\ Yenniel Mendoza** \\ Universidad de La Frontera. Chile \\ https://orcid.org/0000-0001-8144-441X
}

\section{RESUMEN}

En el artículo se exponen los resultados de un estudio sobre la experiencia que tuvo Chile en la creación de Observatorios de turismo en regiones de Chile, considerando dos dimensiones de estudio: en primer lugar, respecto al nivel de desempeño alcanzado atendiendo a sus propósitos basales: monitorear la actividad turística y generar información de ayuda a la toma de decisiones, y en segundo lugar, respecto a la manera en que realizaron la gestión de datos de investigación en turismo, considerando procesos de gestión y apertura. El principal problema evidenciado en los Observatorios de turismo fue la constitución, gestión y difusión de los datos que debían proveer.

Palabras clave: Datos de investigación; información científica; Observatorios en turismo; Turismo.

Fecha de recepción: 29 de junio de 2018.

Fecha de aceptación: 5 de julio de 2019.

* Instituto de Desarrollo Local y Regional, Universidad de La Frontera. Avenida Francisco Salazar 01145. 4811230 TEMUCO (Chile).E-mail: patricio.padilla@ufrontera.cl, sergio.sanchez@ufrontera.cl,j.hernandez15@ ufromail.cl

** Facultad de Ciencias Jurídicas y Empresariales, Universidad de La Frontera. Avenida Francisco Salazar 01145, 4811230, Temuco, Chile.E-mail: yenniel.mendoza@ufrontera.cl

1 Esta investigación corresponde a los resultados del Fondo DIUFRO DI15-0007 "Gestión de datos de investigación en los Observatorios de Turismo en Chile" de la Universidad de La Frontera. Investigador responsable: Patricio Padilla Navarro. 


\title{
Management of research data at the Regional Tourism Observatories in Chile
}

\begin{abstract}
The article presents the results of a study about Chile's experience in the creation of Tourism Observatories in regions of Chile, considering two dimensions of study: firstly, regarding the level of performance achieved according to its basal purposes: To monitor tourism activity and generate information to aid decision-making, and secondly, to the way in which they conducted the management of tourism research data, considering management and opening processes. The main problem evidenced in the Observatories of tourism was the constitution, management and diffusion of the data that they had to provide.
\end{abstract}

Keywords: Research data; Scientific information; Observatories in tourism; Tourism.

\section{INTRODUCCIÓN Y PROBLEMATIZACIÓN}

En Chile se han diseñado diversos programas que buscan generar directrices hacia el desarrollo y posicionamiento del turismo como un eje primordial dentro de la matriz productiva nacional. En el año 2010 se promulgó la ley Nº 20.423 "Del Sistema Institucional para el Desarrollo del Turismo" cuyo objetivo fue el fortalecimiento de la actividad turística, por medio de mecanismos destinados a la creación, conservación y aprovechamiento de los recursos y atractivos turísticos nacionales. El plan de desarrollo turístico chileno 2014 y 2018, consideró el impulso del sector potenciando: i) el desarrollo turístico sustentable en áreas silvestres protegidas del Estado, ii) avanzar en el establecimiento de Zonas de Interés Turístico, iii) impulsar la oferta y promoción del turismo con elementos culturales y iv) facilitar el acceso expedito a instrumentos de fomento a la inversión turística.

Paralelo a esta estrategia de fortalecimiento al sector turístico, Chile se encuentra implementando una política nacional de gestión de datos de investigación e información científica, que ha implicado diagnosticar las capacidades de i) instituciones científicas(universidades, centros de investigación, institutos tecnológicos, núcleos de investigación, entre otros), ii) científicos e investigadores y iii) normativas de fondos de investigación públicos que fomenten la ciencia, tecnología e innovación.

Entre las distintas instituciones científicas que gestionan datos de investigación, los Observatorios son herramientas cuyo quehacer incorpora, en mayor o menor medida, la totalidad de acciones referidas a la gestión de datos, independiente de su área científica o sector de aplicación. Estas acciones de gestión corresponden a la planificación, captura, almacenamiento, procesamiento, validación y diseminación.

En distintas regiones de Chile, durante los años 2007 y 2009 se crearon seis Observatorios de Turismo, pensados como una plataforma para facilitar la gestión de datos de investigación en turismo y así permitir el buen funcionamiento de las políticas de fomento al sector. El objetivo de estos Observatorios fue disponer de información que ayude a la toma de decisiones, pero considerando y complementando los datos de investigación gene- 
rados por dos instituciones públicas vinculadas al turismo en Chile: el Instituto Nacional de Estadísticas (en adelante INE) y el Servicio Nacional de Turismo (en adelante SERNATUR). Sin embargo, existía desconocimiento sobre resultados, impactos, problemáticas y desafíos de estos Observatorios respecto al nivel de cumplimiento de sus propios objetivos y el tipo de gestión de datos realizada. La justificación de estudiar el funcionamiento de estos Observatorios se fundamenta aún más, considerando que dos de ellos fueron cerrados anticipadamente y de los cuatro restantes, solo dos pudieron obtener financiamiento de continuidad. En este sentido, se hizo necesario estudiar los Observatorios de turismo en dos ámbitos: en primer lugar, respecto al nivel de desempeño alcanzado atendiendo a sus propósitos basales: monitorear la actividad turística y generar información de ayuda a la toma de decisiones y, en segundo lugar, respecto a la manera en que realizaron la gestión de datos de investigación, considerando procesos de gestión y apertura.

\section{ANTECEDENTES}

\subsection{Observatorios de turismo en Chile}

En Chile se crearon los siguientes Observatorios de Turismo: i) Observatorio Turístico de la Región de Tarapacá, ii) Observatorio de Turismo Regional de Antofagasta, iii) Observatorio Turístico Región del Maule, iv) Observatorio Turístico Región del Biobío, iv) Observatorio del Sector Turismo, Región de La Araucanía y vi) Observatorio Turístico, Región de Aysén.

Estos Observatorios fueron desarrollados gracias al financiamiento de la Corporación del Fomento a la Producción (en adelante CORFO) a través de su programa Bienes Públicos para la Innovación y Competitividad, cuyo objetivo fue "apoyar proyectos que desarrollen bienes públicos destinados a reducir asimetrías de información, con el propósito de mejorar la competitividad y acelerar el emprendimiento por la vía de disminuir la incertidumbre en la toma de decisiones críticas para el éxito de los participantes y beneficiarios finales del proyecto" (CORFO, 2015:4).CORFO adjudicóen2007 recursos para financiar el Observatorio de la Región de La Araucanía, ejecutado por el Centro de Microdatos de la Universidad de Chile. En 2008se aprobaron recursos para los Observatorios de la Región de Tarapacá, ejecutado por la Universidad Arturo Prat y el Observatorio de Turismo Regional de Antofagasta, ejecutado por la Universidad Católica del Norte. Ambos con el apoyo de las respectivas direcciones regionales de SERNATUR e INE. En 2009 se aprobó el Observatorio Turístico de la Región del Maule, adjudicado a la Universidad de Talca.

El objetivo de estos Observatorios fue generar y proveer periódicamente información detallada, consistente y oportuna sobre la dinámica de la actividad turística, capaz de dimensionar sus niveles e impactos, con la finalidad de anticiparse a los flujos futuros y demandas del sector, constituyendo sistemas de información turística que evidencien las tendencias y características para las respectivas regiones (CORFO y otros, 2013; Observatorio Turístico del Maule, 2012; Agencia Aysén, 2009). Todos los observatorios debían proporcionar informes sobre la situación del turismo regional. Estos informes no tenían un período de lanzamiento determinado para todos los observatorios, pero debían 
ser públicos y considerar tanto análisis estadísticos como reportes de investigación acordados entre las instituciones participantes.

Por otra parte, los Observatorios de la Región del Biobío y de la Región de Aysén fueron financiados por la SUBDERE a través del programa del Fondo Nacional de Desarrollo Regional (en adelante FNDR), cuyo objetivo es fortalecer la capacidad de gestión de los Gobiernos Regionales en materias de inversión pública regional, administrar, controlar y efectuar seguimiento sobre la ejecución financiera de los presupuestos de inversión regional, elaborar y sistematizar información relevante para la toma de decisiones de la autoridad (FNDR, 2016). Ambos Observatorios comenzaron sus funciones el año 2012 y son ejecutados por las direcciones regionales del INE y SERNATUR respectivamente. A diferencia de los Observatorios financiados por la CORFO, estos tienen como objetivo principal implementar un sistema de captura y procesamiento de información confiable, fidedigna y variable sobre la actividad turística, capaz de generar información que permita la toma de decisiones tanto en el sector público como privado, con la finalidad de determinar relaciones y proyecciones dentro del turismo regional (Gobierno Regional Biobío, 2015; Agencia Aysén, 2009).

Actualmente, el Observatorio de la Región de Aysén continúa sus funciones de monitoreo y sistematización de información, ejecutándose bajo el programa denominado "Sistema de Información Turística", financiado a través del FNDR. Además, se indica la pronta firma de un convenio donde se transferirán recursos entre SERNATUR Aysén e INE Aysén, para potenciar las funciones del Observatorio. Por otra parte, el Observatorio de la Región del Biobío concluyó sus funciones el día 30 de mayo de 2015, sin conseguir aprobar su continuidad. Por último, en octubre de 2015, se aprobó un proyecto para la confección de un Observatorio de Turismo en la Región de Los Lagos, adjudicado por la Universidad de Los Lagos y que funcionará durante un periodo de 18 meses $^{2}$.

\subsection{Datos de investigación de turismo en Chile}

Los datos de investigación en turismo en Chile se estructuran en seis grandes categorías que involucran distintas instituciones, repositorios y formas de gestión: i) Ingreso Generado: corresponde al valor de las ventas totales de bienes y servicios relativos al sector turismo a través de las Cuentas Nacionales y la Balanza de pagos. La institución responsable de generar estos datos de investigación es el Banco Central, quien mediante las Cuentas Nacionales permite detectar, para este caso, el aporte de sectores estrechamente vinculados al turismo. ii) Vínculos Intersectoriales: refiere al vínculo que establecen los sectores económicos mediante la demanda de insumos para producir y obtener ingresos. A partir de la Matriz Insumo - Producto se pueden determinar los vínculos directos e indirectos entre los sectores. iii) Empleo Generado y Remuneraciones: a través de los Censos de Población se puede tener información de las personas empleadas en los diferentes sectores, pero es una información que se entrega habitualmente cada 10 años por parte del Instituto Nacional de Estadísticas. Por otra parte, la Encuesta CASEN (Carac-

2 La información de este párrafo fue obtenida mediante una solicitud vía Transparencia al Servicio Nacional del Turismo (Gobierno de Chile) respecto a la continuidad y vigencia de los Observatorios de turismo en Chile que cuenten con apoyo del Instituto Nacional de Estadísticas. 
terización Socioeconómica Nacional), realizada por el Ministerio de Desarrollo Social, también entrega información del empleo por sectores, pero cada 2 o 3 años. iv) Cantidad de Turistas: entre los indicadores turísticos más conocidos están las personas que realizan la actividad turística. En Chile hay varias fuentes de información sobre el flujo de turistas. Por ejemplo, las entradas de extranjeros o las salidas de chilenos al exterior se controlan en todos los pasos oficiales de las fronteras terrestres, en los aeropuertos con vuelos internacionales y en los puertos. Autoridades como la Jefatura Nacional de Extranjería y Policía Internacional de la Policía de Investigaciones de Chile, son los encargados de recoger esta información esencial. v) Días de Estancia: la Encuesta Mensual de Establecimientos de Alojamiento Turístico, del INE, también informa la cantidad de días que están alojados los turistas, tanto nacionales como extranjeros. De esa forma se obtiene el indicador de pernoctaciones, que es la multiplicación de la cantidad de turistas por los días de estancia. vi) Gasto de Turistas: la información sobre el ingreso del sector claramente está vinculado con el gasto que hacen los turistas, pues el flujo circular del ingreso en una economía lleva a que el gasto de un grupo sea a la vez el ingreso de otro grupo. Sin embargo, en este caso es más específica en cuanto a los ingresos que se obtienen a partir de los turistas en actividades de alojamiento. No obstante, hay información más detallada de cuánto gastan los turistas según tipo de alojamiento, temporada y región. vii) Oferta de Servicios Turísticos: en la Encuesta Mensual de Establecimientos de Alojamiento Turístico del INE, se obtiene información detallada de la cantidad de establecimientos por tipos y por región, pero además de la cantidad de establecimientos se puede obtener información más amplia, como es la asociada a la cantidad de habitaciones y de camas, que permite completar el panorama sobre este importante aspecto de la oferta de servicios turísticos. viii) Cuenta Satélite de Turismo: la cuenta satélite de turismo surge de la lógica del sistema de las Cuentas Nacionales. En Chile se realizó en 2003 y a partir de esa fechase han hecho estimaciones posteriores. La cuenta satélite de turismo recoge en esencia toda la información turística, tanto por el lado de la oferta como de la demanda. En esencia, resume en un solo documento toda la información señalada previamente. Se toma en cuenta tanto los flujos monetarios de ingresos y gastos como los flujos de turistas y la disponibilidad de oferta turística y de empleo generado.

\section{MARCO TEÓRICO}

\subsection{Política de gestión de datos de investigación e información científica}

Los datos de investigación se definen como registros de hechos (resultados numéricos, textos, imágenes y sonidos) utilizados como fuentes primarias para la investigación científica y comúnmente aceptados en la comunidad científica, por permitir la validación de resultados. Un conjunto de datos de investigación constituiría una representación sistemática parcial del objeto que está siendo investigado, mientras que la información científica refiere a las conclusiones obtenidas del análisis de datos y a los resultados de una investigación (CONICYT, 2010).A nivel internacional, existen diversos estándares sobre el tratamiento que debiesen recibir tanto datos de investigación como información científica, los que han sido agrupados y promovidos por la OCDE, Unión Europea, el 
G85 y la UNESCO: Apertura, Transparencia, Interoperabilidad, Profesionalismo, Calidad, Eficiencia, Sostenibilidad, Seguridad, Flexibilidad, Conformidad legal y Protección de la propiedad intelectual (Pilat y Fukasaku, 2007). En complemento de ello, también existen diversos estudios y recomendaciones que fomentan al manejo abierto y de libre acceso a los datos de investigación e información científica, basadas en mejorar los procesos de gestión, distribución y promoción de repositorios de datos (Melero y Hernández, 2014),fortalecimiento de bases de datos de instituciones de educación superior, con la finalidad de precisar el tipo de investigación y mejorar los niveles de diseminación(De Feillipo y otros, 2011).

Chile se encuentra desarrollando diversas acciones encaminadas a fortalecer los procesos de gestión de datos de investigación e información en todas las áreas científicas generadas en el país, especialmente las que involucran financiamiento público. Esta necesidad de fortalecimiento institucional nace fundamentalmente de dos procesos que orientan y sugieren al país cómo afrontar el desafío de mejorar los niveles de gestión de datos e información. En primer lugar, se encuentra un estudio sobre el estado del arte nacional e internacional en materia de gestión de datos de investigación e información científica,recomendaciones de buenas prácticas y un manual de datos abiertos. En segundo lugar,unManualque explica la política de datos abiertos y entrega recomendaciones para el acceso y preservación de información científica y datos de investigación. A continuación, se explican ambos procesos.

\subsubsection{Estado del arte y recomendaciones de buenas prácticas}

En 2010 se realizó un estudio con el estado del arte nacional e internacional en materia de gestión de datos de investigación e información científica, extrayendo recomendaciones de buenas prácticas y aprendizajes para el caso chileno(Padilla y otros, 2013; Sanhueza y otros, 2012; CONICYT, 2010). El estudio constató que:i) Tanto las instituciones científicas como los investigadores tienen un rol pasivo en la diseminación del patrimonio de datos e información financiados con fondos públicos. A pesar de que existe la percepción sobre la importancia de dar acceso a la ciencia, las acciones de intercambio en Chile no corresponden a procesos de diseminación considerando buenas prácticas internacionales. ii) Los fondos públicos de fomento a la ciencia en Chile no definen la responsabilidad respecto al tratamiento de los datos e información generados, tanto en tecnologías, formatos, como las propias acciones de difusión y diseminación. iii) Existe una importante brecha tecnológica, ya que la gestión de datos e información se realiza con ausencia de manuales, administradores, protocolos de respaldo y bases de datos especializadas. iv) Se relevó la necesidad de impulsar el carácter público de la ciencia financiada con fondos provenientes de servicios del Estado, para propiciar una participación en prácticas de diseminación de datos e información. v)Se constató la necesidad de resguardar diferenciadamente el patrimonio de datos e información, que merezcan un trato especial por su carácter estratégico, ya sea por motivos de seguridad nacional o riesgos que impliquen su mala utilización. Esto se traduce en disponer de protocolos especiales y establecer claramente los motivos para restringir el acceso. vi) Finalmente, una de las recomendaciones estableció el fortalecimiento a la transdisci- 
plina en la gestión de datos e información, dado la diversidad de tareas en materia de captura, almacenamiento, procesamiento y diseminación, donde interceden gestores de información, ingenieros informáticos, lingüistas, bibliotecólogos, entre otros.

\subsubsection{Manual de datos abiertos}

Considerando los resultados del estudio anterior, en 2014 se realizó un manual de datos abiertos que reúne orientaciones y directrices elaboradas por la OCDE, acuerdos de la Unión Europea, el G85 y la UNESCO(CONICYT, 2014). El manual ofrece recomendaciones para fortalecer los procesos de acceso a datos de investigación e información científica. En información científica se recomienda a la comunidad científica utilizar licencias abiertas, recurrir a especialistas en gestión de información, creación de repositorios institucionales y utilizar repositorios interoperables. Para datos de investigación también se sugiere utilizar licencias abiertas y recurrir a asesorías, pero adicionalmente se recomienda mejorar las descripciones e identificación en los datos, asegurar la perdurabilidad del patrimonio de datos y utilizar bases de datos especializadas.

Una tercera iniciativa que se desprende de las anteriores refiere a la generación de estudios específicos que permitan mejorar los procedimientos en gestión de datos e información para determinadas áreas científicas o instituciones, cuyo quehacer implique la gestión de distintos tipos de datos e información. Ejemplo de esto último, fueron los problemas de coordinación e interoperabilidad en el terremoto del 27 de febrero de 2010 en Chile, donde parte de los problemas que existieron para abordar de mejor manera esta catástrofe, fue la descoordinación en el tratamiento de datos oceanográficos, vulcanológicos y sismológicos por parte de la institucionalidad chilena (Cancino y Seguel, 2014).En este sentido, los aspectos relativos al carácter público cobrarían una relevancia adicional en materias estratégicas y/o de seguridad nacional (Uhlir y Schröder, 2007) pero a la vez exigirían determinados tipos de controles y restricciones de uso. Por ejemplo, algunos datos no se pueden entregar de forma aislada, siendo necesario dejarlos a disposición con otros datos adicionales (conjuntos de datos, datasets) proceso que puede revestir complejidades dependiendo del carácter de la información en cuestión. En este sentido, se recomienda incorporar resguardos para asegurar que la utilización de esos datos sea correcta, no se lucre con datos provistos por fondos públicos y no exista manipulación de información. Adicionalmente, algunos requerimientos de interoperabilidad de datos implican restricciones éticas que limitan o prohíben el acceso a información de carácter personal (Mendo y otros, 2013).Las restricciones más frecuentes se relacionan con el tipo de financiamiento de las investigaciones; por ejemplo, la participación de políticas de acceso abierto por parte de universidades públicas en España, se relaciona fundamentalmente por el carácter estatal del financiamiento en investigaciones científicas, que en gran medida reglamentan la manera en cómo se almacenan y distribuyen los datos provenientes de dichas investigaciones (Abadal y otros, 2013), aspecto que en el caso chileno no tendría el mismo nivel de regulación, dado que los fondos públicos orientados al fomento de la ciencia y tecnología poseerían ambigüedades a la hora de definir responsabilidades en la gestión de datos de investigación e información científica (Sanhueza, Padilla, Rodríguez, 2012). 


\subsection{Conformación de datos de investigación de turismo}

Parte de la discusión sobre el turismo como objeto de estudio, ha estado centrada en otorgar al turismo de una episteme y de conceptualizaciones que permitan dimensionarlo no sólo con variables cuantitativas y análisis de mercados, sino como un cuerpo teórico con densidad propia y delimitada. Ejemplos de esta discusión corresponden a la inclusión de ejes de análisis como el Tiempo, Espacio, Motivaciones y Actividades (T.E.M.A.) que permitirían dar cuenta de los distintos procesos del fenómeno turístico, articulando los componentes objetivos y subjetivos, permitiendo investigaciones multidimensionales (Campodónico y Chalar, 2011), así como la propuesta de generar una ruptura epistemológica -con cierto compromiso teórico-que abogue por un turismo enfocado al estudio de relaciones humanas y contribuya a convertir las prácticas sociales del turismo, en más responsables, sostenibles y por consiguiente, más humanas (Martini Moesch, 2013).

La literatura sobre gestión de datos de investigación se ha concentrado en las características de la diseminación en la investigación del turismo, centrándose en países, instituciones, investigadores y revistas de investigación turística en Iberoamérica. Por ejemplo, para la productividad científica en turismo de los países iberoamericanos, destacan Brasil, España y en tercer lugar México, país que ha mantenido un elevado nivel de publicaciones desde 2009, con 25 publicaciones anuales. El caso mexicano se caracteriza porque su producción científica en turismo concentra el $63 \%$ en revistas latinoamericanas por sobre las revistas brasileñas, españolas o internacionales de habla inglesa (Picazo y Moreno, 2013). Por su parte, la evolución de Argentina y Chile presenta un crecimiento intermitente hasta el año 2010 y comienza a disminuir en 2011 ,evidenciándose un mayor volumen de publicaciones por parte de los investigadores argentinos (Picazo y Moreno, 2012).

Como sector económico, el turismo ha experimentado un crecimiento sostenido durante las últimas décadas, contribuyendo a la economía a nivel mundial, mediante la creación de empleos, empresas, emprendimientos, infraestructura y ganancias de exportaciones, tanto para las economías avanzadas como para las emergentes (MMA, 2014). Considerando lo anterior, existiría una demanda creciente sobre información relativa al turismo por parte de sectores empresariales y servicios públicos vinculados al turismo, pero que se complejizaría por la diversidad en las fuentes de información, donde cada usuario demandaría un tipo de información específica, resultando necesario disponer de sistemas de información especializados en el sector. Por otra parte, a la complejidad que envuelve el turismo, involucrando a todas las actividades económicas, los distintos niveles de gobierno y los sectores público y privados (Velarde-Valdez y otros, 2015), se debe incluir el interés de comunidades y órganos públicos municipales, centrados principalmente en el desarrollo económico de ciertas localidades (Biz y otros, 2011). Esto ha fomento la creación de una plataforma institucional que acompaña al sector en distintos ámbitos. Particularmente, García Hernández, en el marco de un estudio sobre las ciudades patrimonio de la humanidad de España, identificó distintas configuraciones institucionales que permiten planificar y gestionar el turismo. Entre ellas se encontraron las Concejalías en Turismo, organismos autónomos de Gestión turística, organismos con funciones específicas respecto a segmentos de mercado turístico, consorcios con competencias en la conservación y recuperación patrimonial y las estructuras supralocales de gestión conjunta (2007). 
Jafari (2005), en un diagnóstico general sobre el turismo respecto a su avance y consolidación como disciplina científica, profundiza en los desafíos que devienen para afrontar el futuro, argumentando que es momento de cambiar la actitud reactiva que tienen gobernantes y tecnócratas frente al turismo. Paralelamente, la globalidad en que se desenvuelve el turismo implicaría que ningún país, región o localidad quede excluida. Por ende, gestionar información turística contingente y desarrollar la investigación dentro del campo se vuelve una necesidad. Para ambas situaciones, Jafari plantea la constitución de Observatoriosde Turismo (OT), los que se caracterizarían por su capacidad anticipadora, pues deben contar con una amplia base asociativa que incluya representación del sector público y privado, considerando agencias gubernamentales, empresas operativas, instituciones académicas y asociaciones ciudadanas. Además, deberían abarcar y conocer el conjunto de elementos relevantes, geopolíticos, socioeconómicos y ambientales (así como las fuerzas tradicionales del mercado) que afectan a su destino y prevé el futuro, incluyendo lo probable (preparándose para ello en consecuencia) y lo que lo es menos, es decir, las hipótesis alternativas (diseñando los planes correspondientes) (Jafari, 2005:53).

Estos Observatorios serían esenciales a la hora de planificar la labor turística en las zonas donde se encuentran operando, pues se transforman en informantes coyunturales, los que proporcionarían información eficiente que ayudaría a la toma de decisiones y al desarrollo del planeamiento en turismo. Además, el generar información respecto a condiciones sociales, culturales y naturales, tendría importancia para visibilizar lugares, zonas o hitos con potencial turístico. Para Jafari, esta información estaría siendo proporcionada por los distintos actores inmersos en el sector, como turistas y residentes, así como también del ámbito productivo integrado por empresas e instituciones del sector público. Sin embargo, el carácter de esta información sería mayormente exploratoria y descriptiva, no existiendo organismos especializados dedicados específicamente al análisis, diagnóstico y elaboración de programas para el fomento y el mejoramiento de las condiciones de la actividad turística (Jafari, 2005).

Enrique Torres Bernier (citado en Santágata, 2011) plantea 4 puntos específicos respecto a la constitución y funciones de un Observatorio de Turismo: (1) un instrumento que ayuda a la toma de decisiones de la iniciativa privada (empresarios) y pública (administraciones) de un territorio que se identifica como un destino turístico, real o potencial; (2) un instrumento para controlar los resultados de las políticas turísticas y ayudar a su posible corrección; (3) una base para la elaboración de diagnósticos e informes de los destinos turísticos a los que se aplica; (4) una base para la elaboración de modelos de predicción de las principales variables turísticas a corto plazo. Así mismo, las labores de planificación que competen a los Observatorios se pueden sintetizar en la realización de investigación sistémica (de la región o zona) que proporcione los elementos necesarios para el diagnóstico, que servirá de base para la planificación participativa, la que determinará las características necesarias para la implementación de políticas en turismo. Finalmente está el control, que constatará el cumplimiento o no de las acciones propuestas (Santágata, 2011). Ejemplos de ello ha sido la creación del Centro de Investigación en Turismo y Patrimonio de la Región de Valparaíso en Chile (González y otros, 2013) y el Observatorio de Turismo en Ecuador (Burneo y López, 2013) ya que ambas experiencias tuvieron que combinar 
requerimientos basados en la investigación sobre el turismo con demandas vinculadas al sector productivo y a la institucionalidad pública de fortalecimiento al turismo en sus respectivas zonas de ejecución.

\section{MATERIALES Y MÉTODOS}

\subsection{Enfoque y propósito}

Se trata de una investigación de corte principalmente cuantitativo, con nivel de alcance descriptivo, que opera en base a la utilización de metodologías de encuesta online a informantes claves.

La investigación pretendió describir la constitución, ejecución y desarrollo de los Observatorios en las regiones de Chile, con énfasis en el cumplimiento de los objetivos y de los compromisos establecidos. Por otra parte, se buscó conocer la calidad y la competencia de los datos generados y su correspondencia con estándares internacionales.

\subsection{Muestra}

Para este caso no se definió un diseño muestral que implique el cálculo de una muestra, ni la estimación del error y/o la selección aleatoria de las unidades para la estimación de parámetros. En esta ocasión, los resultados estuvieron referidos a la población. Bajo esta premisa - la de la no generalización de los resultados- la investigación corresponde más a un estudio de caso o un muestreo de tipo intencional, que a un diseño probabilístico o inferencial.

La población de estudio se definió como "Los Observatorios de Turismo Regional en Chile que operaron entre los años 2007 y 2009". Seis son los Observatorios que respondieron a esta definición, los cuales fueron ejecutados en las regiones de Tarapacá, Antofagasta, Maule, Biobío, La Araucanía y Aysén. Además de los Observatorios señalados, se consideró en la selección de los casos a las direcciones regionales del INE y SERNATUR, instituciones que participaron en el desarrollo de este proyecto. De esta forma, se aplicaron un total de 18 encuestas, 3 por cada región: 1 al directorio de cada Observatorio Regional, 1 al directorio del SERNATUR de cada región y 1 al directorio del INE regional respectivo. En este caso, la selección consideró que el encuestado haya sido director o coordinador de los observatorios contemplados en el estudio.

\subsection{Dimensiones y variables}

Las dimensiones de análisis estuvieron asociadas a la definición de los objetivos específicos de la investigación: por un lado, evaluar el cumplimiento de objetivos y, por otro lado, el proceso de generación de datos. En términos específicos, el proceso de definición de dimensiones y operacionalización de variables dio como resultado una matriz compuesta por 2 dimensiones y 7 variables que se encuentran en la tabla 1 . 


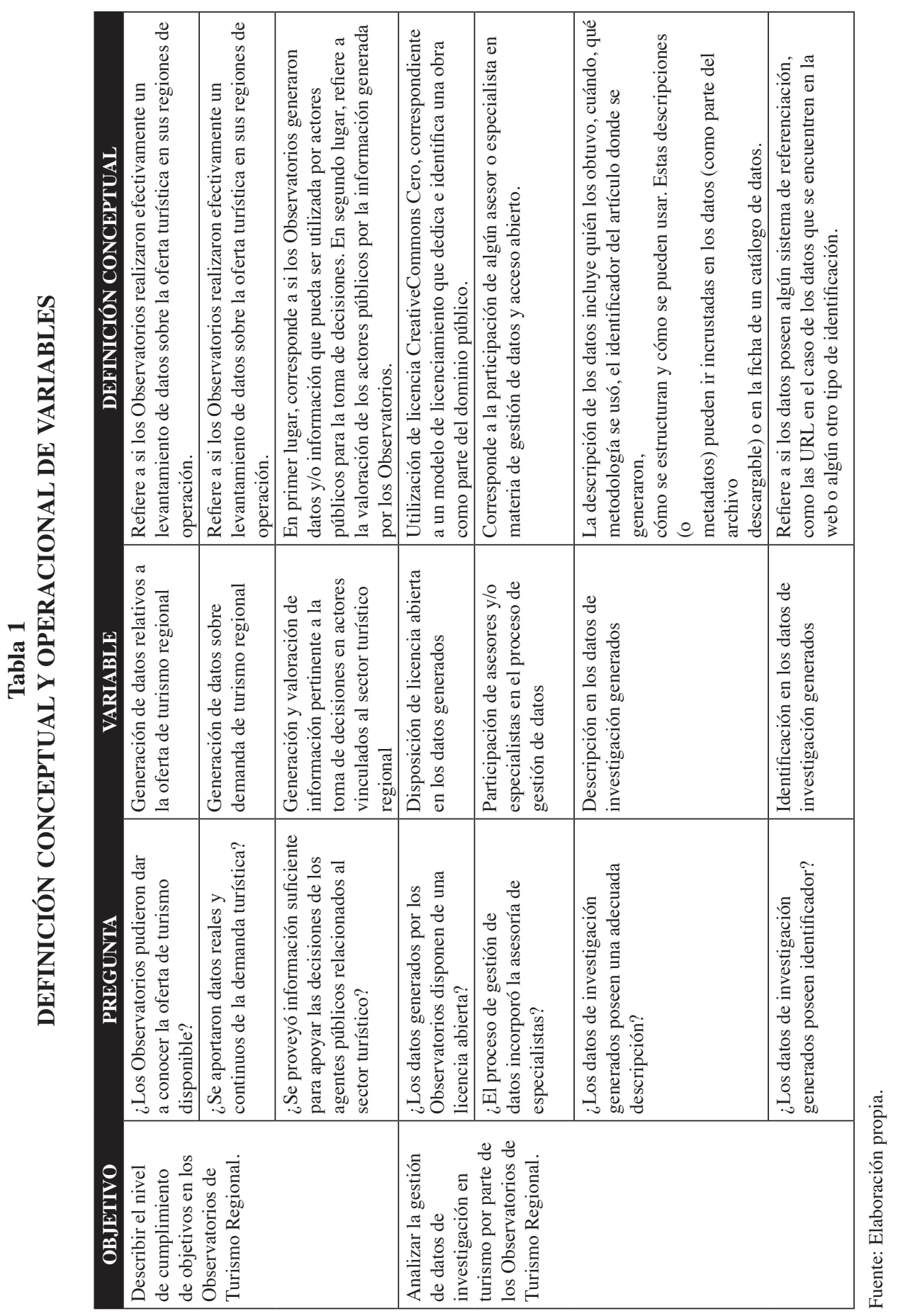




\subsection{Técnicas de recolección de información}

La investigación estuvo basada principalmente en el uso de Metodologías de Encuesta y específicamente en el uso de Metodologías de Encuesta Online. En la actualidad, el uso de este tipo de herramientas es fundamental, en la medida que permiten adaptarse a las nuevas formas de comunicación, reducir los costos y aumentar el nivel de control del proceso de aplicación. Complementariamente, se utilizaron técnicas de Análisis Documental en fuentes de información secundaria ("información gris"), como términos de referencia de los concursos para los Observatorios, actas de apertura y cierre, informes técnicos, entre otros, los que permitieron reconstruir el contexto en el que se desarrolló el proyecto.

El instrumento propuesto estuvo conformado en su mayoría por sets de preguntas cerradas, con una estructura de respuesta ordinal en 3 niveles (bajo, medio, alto), lo que posibilita en gran medida la comparación de variables y la construcción de índices sintéticos o agregados. En la lógica de obtener información cualitativa, se consideró también una pregunta abierta por cada dimensión, orientada a la identificación de los factores o las causas del desempeño de los Observatorios.

\subsection{Técnicas de análisis de datos}

Dado que la investigación tuvo como propósito lograr una primera aproximación al caso de los Observatorios Regionales de Turismo en Chile, los análisis fueron fundamentalmente descriptivos.

Para el caso de variables consultadas a través de técnicas de encuesta, se han llevado a cabo análisis de las distribuciones de frecuencias y estadísticos de tendencia central. Cabe señalar que, al tratarse de un número reducido de casos, los análisis no han estado centrados en la descripción de los porcentajes de las categorías, sino más bien en las tendencias generales de los datos. Con lo anterior, se han construido también dos índices sintéticos (uno por dimensión): uno referido al nivel de desempeño de los Observatorios y otro al proceso de gestión de datos, lo que permite una aproximación más global a las dimensiones estudiadas.

\section{6. Índices y clasificaciones}

Con el propósito de establecer comparaciones y específicamente agregar información en índices de mayor valor analítico, se planteó el diseño de la mayoría de las variables del instrumento, en un nivel de medida ordinal con 3 categorías de respuesta, que permitieran describir niveles: bajos, intermedios o altos de desempeño. Para el cálculo de los respectivos índices, se procedió a estimar la media de los puntajes obtenidos (y no la sumatoria de los puntajes), de tal forma que la interpretación de los resultados fuera más gráfica y acorde a la escala de respuesta del cuestionario.

Considerando que, una vez agregadas las variables en un índice sintético, se obtiene como resultado una variable continua (con valores intermedios o decimales), la construcción de la escala de percepción del desempeño, se estableció fijando a priori 4 puntos de corte en un intervalo de 1 a 3 . Los puntos de corte fueron: 1,40 - 1,80 - 2,20 y 2,60. Con 
ello se buscaba disponer de cinco categorías que permitieran describir distintos niveles de desempeño alcanzado, las que fueron definidas como: muy mala percepción, mala percepción, regular percepción, buena percepción y muy buena percepción ${ }^{3}$.

De esta forma, la escala de percepción, donde 1 representa el intervalo de menor desempeño y 5 el intervalo de mayor desempeño, quedó expresada de la siguiente forma:

1.Muy mala percepción sobre el desempeño: $1,00-1,39$

2.Mala percepción sobre el desempeño: $1,40-1,79$

3. Regular percepción sobre el desempeño: 1,80-2,19

4.Buena percepción sobre el desempeño: 2,20 - 2,59

5.Muy buena percepción sobre el desempeño: 2,60 - 3,00

Además, de acuerdo con el desarrollo de sus funciones, los Observatorios fueron agrupados en tres tipos:

a.Finalización Anticipada: Observatorios que debido a malos resultados debieron cerrarse antes que concluyera su financiamiento basal

b.Finalización Normal: Observatorios que cumplieron con el tiempo de ejecución y financiamiento basal pero que no continuaron tras finalizar el proyecto

c. En Funcionamiento: Observatorios que continúan en ejecución debido a sus buenos resultados y a la obtención de financiamiento adicional.

\section{RESULTADOS}

\subsection{Resultados globales}

En este primer apartado de resultados se revisará el nivel de cumplimiento de objetivos y la gestión de datos de investigación en los Observatorios de Turismo Regional, considerando la percepción de los directores de Servicio del INE y SERNATUR a nivel regional. Los resultados sobre cumplimiento de objetivos y gestión de datos de investigación pueden sintetizarse en la tabla 2.

\section{Tabla 2 \\ SÍNTESIS DE RESULTADOS}

\begin{tabular}{|c|c|}
\hline \multicolumn{2}{|c|}{ CUMPLIMIENTO DE OBJETIVOS } \\
\hline ÍTEM & RESULTADOS MAYORITARIOS \\
\hline $\begin{array}{c}\text { ¿Los Observatorios pudieron dar a conocer la oferta } \\
\text { turística disponible?: }\end{array}$ & Se dio a conocer medianamente \\
\hline $\begin{array}{c}\text { ¿Se aportaron datos empíricos y permanentes sobre la } \\
\text { demanda turística? }\end{array}$ & $\begin{array}{c}\text { Se aportaron / Se aportaron } \\
\text { medianamente }\end{array}$ \\
\hline
\end{tabular}

3 Lo anterior es un procedimiento utilizado a menudo en el trabajo con escalas actitudinales. En este caso, para la construcción de la escala de valoración del desempeño, no se implementaron procedimientos estadísticos avanzados para el estudio de las distribuciones, que permitiera establecer matemáticamente puntos de corte a partir de la forma en que se agrupen los datos. 


\begin{tabular}{|c|c|}
\hline \multicolumn{2}{|c|}{ CUMPLIMIENTO DE OBJETIVOS } \\
\hline ÍTEM & RESULTADOS MAYORITARIOS \\
\hline $\begin{array}{c}\text { ¿Se proveyó la información suficiente para la } \\
\text { toma decisiones por parte de los actores públicos } \\
\text { vinculados al sector turismo? }\end{array}$ & Se proveyó medianamente \\
\hline \multicolumn{2}{|c|}{ GESTIÓN DE DATOS DE INVESTIGACIÓN } \\
\hline $\begin{array}{c}\text { ÍTEM } \\
\text { ¿Los datos generados por los Observatorios disponen } \\
\text { de licencia abierta Creative Commons? }\end{array}$ & RESULTADOS MAYORITARIOS \\
\hline $\begin{array}{c}\text { ¿El proceso de gestión de datos incluyó la asesoría de } \\
\text { especialistas? }\end{array}$ & Se incluyeron / Se desconoce \\
\hline ¿Los datos generados poseen una adecuada \\
descripción?
\end{tabular}

Fuente: Elaboración propia.

Respecto al cumplimiento de objetivos, la percepción mayoritaria sobre el quehacer de los Observatorios es que proporcionaron medianamente los datos de la oferta turística regional, siendo reducidas las opiniones favorables sobre la gestión de los Observatorios regionales en este aspecto.

En segundo lugar, existieron dos grandes grupos de percepciones respecto al aporte que hicieron los Observatorios en datos empíricos y permanentes sobre la demanda turística: un grupo que consideró relevante este aporte y otro que lo catalogó como un aporte mediano.

En tercer lugar, la mayor parte de los encuestados señaló que los Observatorios proporcionaron medianamente información para la toma decisiones de actores públicos vinculados al sector turismo.

Frente a los aspectos que dificultaron el cumplimiento de los objetivos por parte del Observatorio Regional de Turismo, las percepciones apuntaron hacia aspectos más enfocados a descoordinaciones institucionales, tales como: a) No habría existido claridad de los roles que debían cumplir el SERNATUR y el INE respecto al funcionamiento de los Observatorios. b) El proyecto de Observatorios no habría tenido objetivos claros ni experiencias previas en las cuales basar una gestión a nivel regional. c) Los Observatorios habrían tenido una escasa participación de las contrapartes a nivel local, específicamente de SERNATUR regional y las Universidades regionales, ya que la concentración de las decisiones habría estado en SERNATUR Chile. d) En el tiempo de ejecución de los Observatorios, habría existido una excesiva rotación de directores en el SERNATUR y de contrapartes vinculadas al proyecto. e) Los proyectos de Observatorios habrían utilizado un tiempo excesivo para iniciar su funcionamiento. 
Respecto a la gestión de datos de investigación realizada por los Observatorios, en primer lugar, existió un mayoritario desconocimiento sobre si los datos generados por los Observatorios dispusieron o no de licencias abiertas Creative Commons. Cabe recordar que la utilidad de estas licencias es regular los derechos de uso de los datos por otros actores e instituciones.

En segundo lugar, los resultados arrojaron que la incorporación de especialistas en el proceso de gestión de datos en los Observatorios tuvo dos grandes tipos de percepciones: los Observatorios que incluyeron un especialista de gestión de datos y los que desconocen si un profesional de estas características fue incorporado al equipo.

En tercer lugar, respecto a las características de los datos generados por los Observatorios, los resultados arrojaron que los datos, sólo en algunos casos, tuvieron una descripción de datos (o metadatos), cuya utilidad es indicar el contenido de los datos, quién los obtuvo, cuándo, qué metodología se usó, el identificador del artículo donde se generaron, cómo se estructuraron y cómo pueden ser utilizados. Junto con ello, igualmente sólo en algunos casos los datos generados por los Observatorios tuvieron un identificador de datos, cuya utilidad radica en permitir que el dato se referencie, tanto en el artículo para el que se generaron como en artículos de otros investigadores que reutilicen esos datos.

Finalmente, los aspectos que los directores del SERNATUR e INE regionales señalaron como obstáculos en la gestión de datos fueron los siguientes: a) Habría existido un problema en las mediciones, dado que se habrían realizado por provincia cuando los datos tendrían un carácter regional, no existiendo desagregación de destinos, ya que los Observatorios se abocaron solamente en estudiar perfiles y en la caracterización de los visitantes. b) Inexistencia de especialistas en gestión de datos de investigación en turismo que pudiesen apoyar el quehacer metodológico de los Observatorios.

\section{2. Índice de percepción de desempeño}

Considerando los datos analizados y en función de la escalas y las categorías propuestas en el apartado anterior, es posible señalar que la Percepción sobre los Observatorios de Turismo en Chile alcanzó un desempeño Regular. Por su parte, las Direcciones de INE percibieron el Desempeño de los Observatorios de manera Buena, mientras que las Direcciones del SERNATUR lo percibieron de manera Regular. Asimismo, los Observatorios que tuvieron "Finalización Anticipada" y "Finalización Normal" obtuvieron una percepción Regular sobre su desempeño, mientras que los que continúan "En Funcionamiento" obtuvieron una Buena evaluación (ver gráfico 1). 


\section{Gráfico 1 \\ PERCEPCIÓN DE DESEMPEÑO GENERAL}

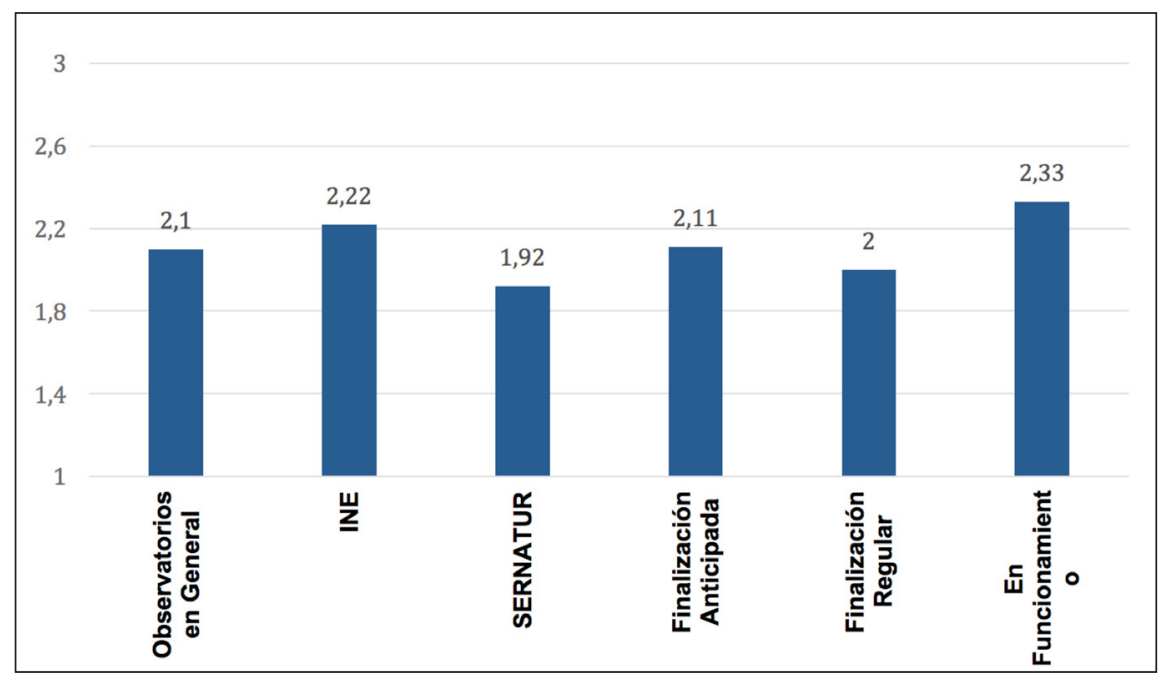

Fuente: Elaboración propia.

\subsubsection{Ejecutores y Co-Ejecutores}

Los ejecutores de los Observatorios se clasificaron en dos tipos: universidades e instituciones públicas (INE y SERNATUR). La percepción en general para los Observatorios de Turismo ejecutados, tanto por universidades como por instituciones públicas, fue Regular, con 2,17 y 2,00 puntos respectivamente (ver gráfico 2).

Los co-ejecutores tuvieron la misma clasificación (universidades e instituciones públicas), salvo el caso del Observatorio de la Región de La Araucanía, donde se identificó como co-ejecutor una entidad de derecho privado: CorpAraucanía. El desempeño de los Observatorios co-ejecutados por universidades fue percibido como Bueno, mientras que para los Observatorios co-ejecutados por instituciones públicas, su desempeño fue Regular (ver gráfico 2).

En ambos casos, el desempeño de los Observatorios desarrollados por universidades fue percibido de mejor manera, respecto de las instituciones públicas. Sin embargo, cabe mencionar que los Observatorios desarrollados entre universidades e instituciones públicas tuvieron un desempeño Regular, al igual que los Observatorios desarrollados sólo por instituciones públicas, con 2,08 y 2,00 puntos respectivamente. Por último, los Observatorios desarrollados sólo por universidades obtuvieron un desempeño Bueno, con 2,33 puntos.

Considerando los perfiles anteriores, cabe destacar que dos de los tres Observatorios que fueron desarrollados entre universidades e instituciones públicas, tuvieron Finalización Anticipada, mientras que el Observatorio ejecutado y co-ejecutado sólo por universidades tuvo Finalización Normal. Asimismo, uno de los dos Observatorios desarrollados exclusivamente por Instituciones Públicas se encuentra En Funcionamiento. 
PERCEPCIÓN DE DESEMPEÑO EN EJECUTORES Y CO-EJECUTORES

3

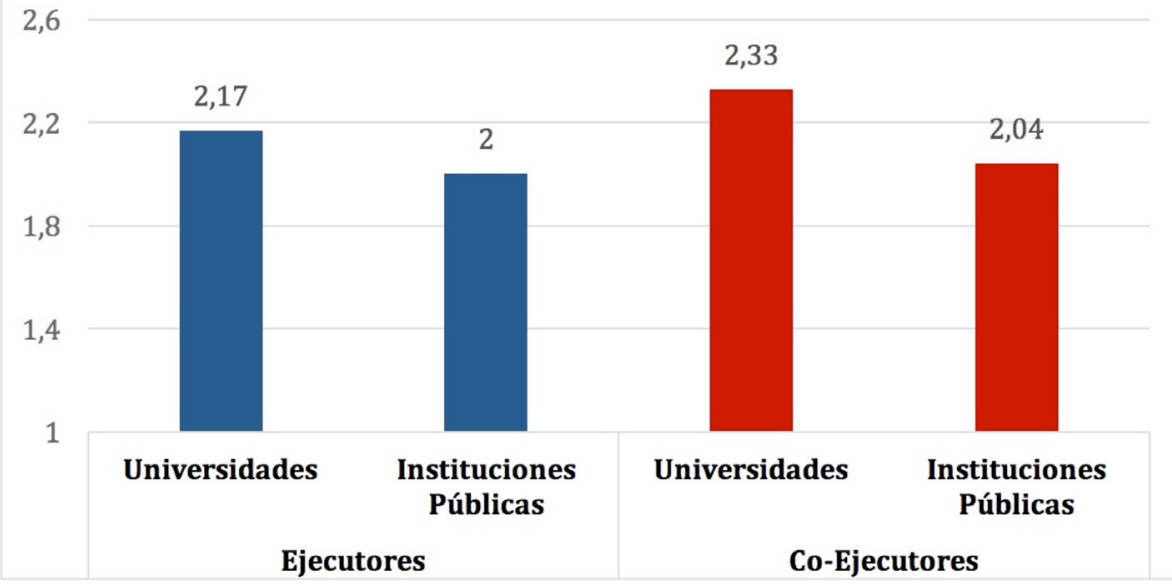

Fuente: Elaboración propia.

Gráfico 3

PERCEPCIÓN DE DESEMPEÑO EN INSTITUCIONES

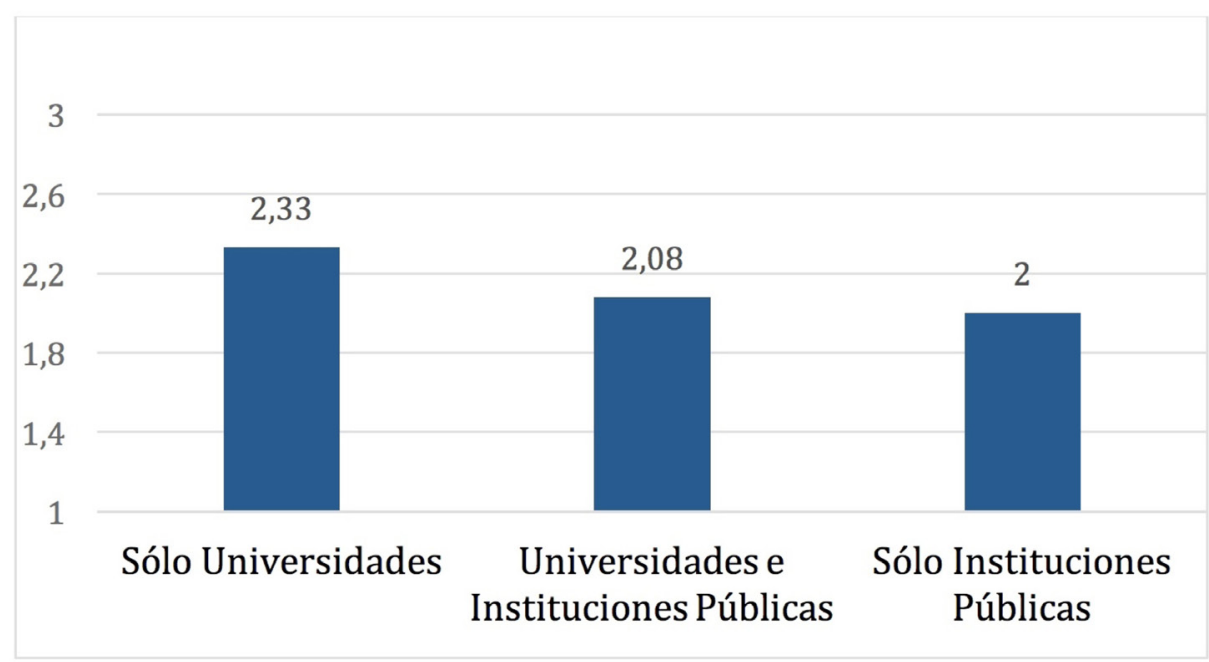

Fuente: Elaboración propia. 


\subsubsection{Líneas de Financiamiento y Montos Basales}

Respecto a los medios de financiamiento que tuvieron cada uno de los Observatorios, cuatro fueron financiados a través de presupuesto asignado por Innova-CORFO y dos a través del FNDR. Para ambas líneas de financiamiento, la percepción sobre el desempeño fue Regular, con 2,17 y 2,0 puntos respectivamente.

De acuerdo con los montos basales, el Observatorio de la Región de Antofagasta fue el que obtuvo menor financiamiento, con un monto de 214 millones de pesos provenientes de Innova-CORFO. Este Observatorio fue ejecutado por la Universidad Católica del Norte y co-ejecutado por las direcciones regionales de INE y SERNATUR. A pesar de que la percepción sobre su desempeño fue Buena, este Observatorio tuvo una Finalización Anticipada.

El Observatorio de la Región del Biobío, obtuvo un financiamiento del orden de 218 millones, entregados por el FNDR. Este Observatorio fue ejecutado por la dirección regional del INE y co-ejecutado por la dirección regional del SERNATUR. Dicho Observatorio, tuvo una Finalización Normal y la percepción sobre su Desempeño fue Mala, con 1,67 puntos.

En tercer lugar, se encuentra el Observatorio de la Región de Tarapacá, el que obtuvo un monto basal de 254 millones asignados por Innova-CORFO. Este Observatorio fue ejecutado por la Universidad Arturo Prat y co-ejecutado por la dirección regional del INE, siendo su mandante el SERNATUR Regional. Este Observatorio tuvo un Finalización Normal y la percepción de su desempeño fue Regular, con 2,00 puntos.

Le sigue el Observatorio de la Región del Maule, con un monto basal de 256 millones, asignados por Innova-CORFO. Este Observatorio fue ejecutado por la Universidad de Talca y co-ejecutado por la Universidad Austral de Chile, proyecto que tuvo Finalización Normal y la Percepción sobre su Desempeño fue Buena, con 2,33 puntos.

El segundo monto más alto lo obtiene el Observatorio de la Región de La Araucanía, con un monto basal de 317 millones, entregados por Innova-CORFO. Este Observatorio fue ejecutado por el Centro de Micro-Datos de la Universidad de Chile y Co-Ejecutado por las direcciones regionales de INE, SERNATUR y CorpAraucanía. A pesar de obtener uno de los más altos montos basales, este Observatorio tuvo una Finalización Anticipada, siendo el peor valorado con un desempeño: Muy Malo, con 1,33 puntos.

Por último, el Observatorio de la Región de Aysén fue el que obtuvo el monto basal más alto, con un total de 350 millones, asignados por el FNDR. Este Observatorio fue ejecutado por la dirección regional del SERNATUR y co-ejecutado por la dirección regional del INE. Actualmente continúa En Funcionamiento y hubo una Buena percepción sobre su desempeño (2,33 puntos). 


\section{Gráfico 4 \\ PERCEPCIÓN DE DESEMPEÑO Y FINANCIAMIENTO DE LOS OBSERVATO- RIOS DE TURISMO}

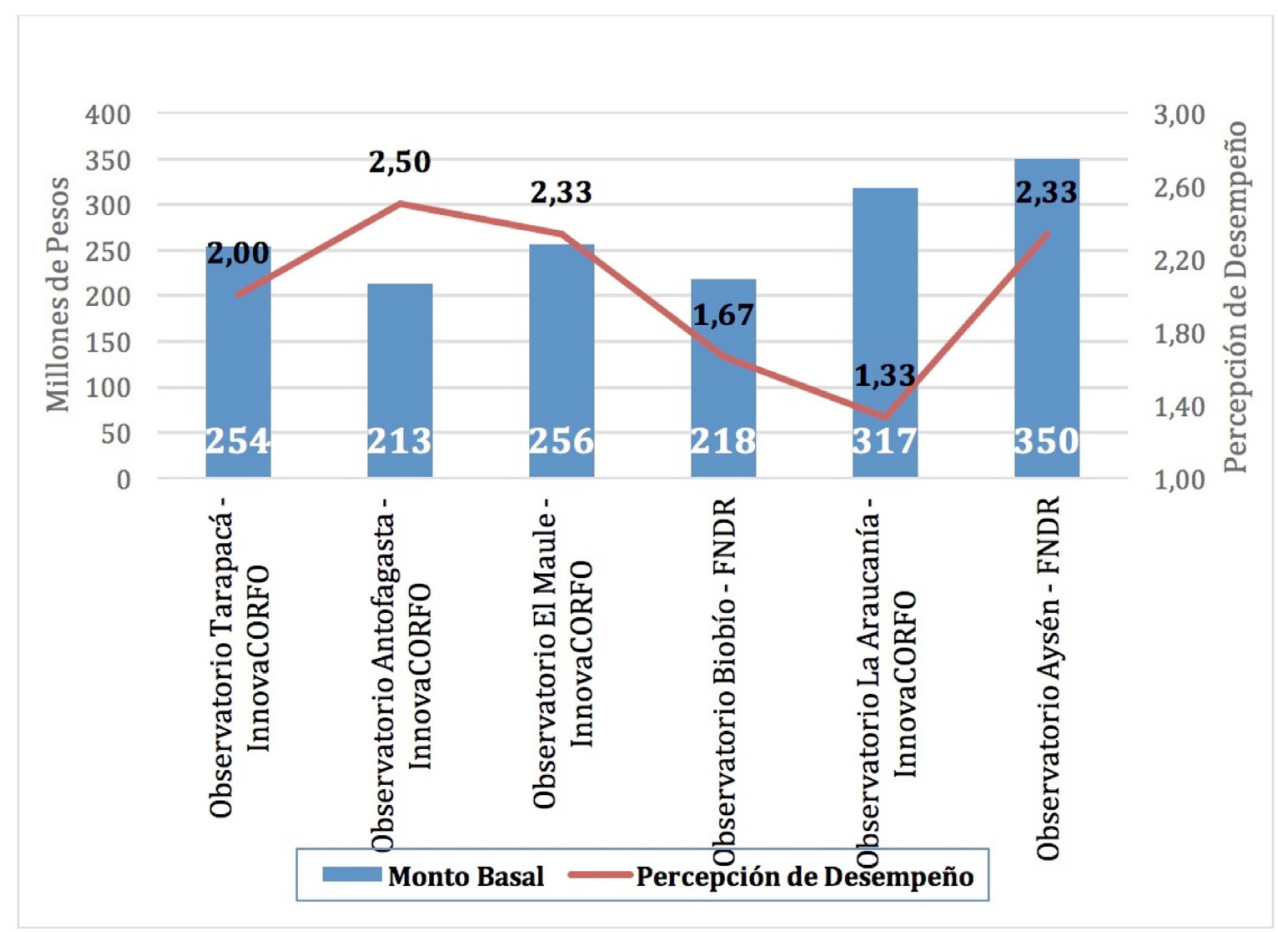

Fuente: Elaboración propia.

\section{DISCUSIÓN Y CONCLUSIONES}

En base a los resultados obtenidos, el principal problema evidenciado en los Observatorios de turismo se relacionó a la constitución, gestión y difusión de los datos que debían proveer. Paralelamente, no necesariamente el desempeño de los Observatorios estuvo supeditado en aspectos exclusivamente económicos, pues como se estableció en los resultados, no existió una clara correlación entre los montos basales y el desarrollo de las funciones. Por un lado, el Observatorio de la región de Antofagasta fue el que recibió menos financiamiento, y por otro, el Observatorio de la Región de La Araucanía fue el segundo en recibir un monto de aprobación alto, considerando que ambos tuvieron un cierre anticipado de sus funciones.

La construcción del Índice de Percepción de Desempeño, ofreció una serie de ventajas para el análisis de los datos. En primer, lugar permitió sintetizar de mejor forma la información obtenida y aproximarse desde una perspectiva más global a las dimensiones de análisis. Al agrupar un mayor número de variables, fue posible la obtención de resultados de mayor valor analítico o de variables con mayores propiedades. En este caso particular, 
permitió conocer si factores como la entidad ejecutora o fuente de financiamiento, explicaban de cierta forma la percepción del nivel de desempeño de los Observatorios.

La coordinación institucional fue uno de los mayores problemas en la gestión de los observatorios, tanto por la escasa participación de sus instituciones participantes como de la dificultad para identificar objetivos claros para su accionar. Sumado a lo anterior, la rotación de directores y asimetrías en el peso de las decisiones también contribuyó al regular desempeño que tuvieron. Sin embargo, es necesario destacar que los observatorios ejecutados por universidades tuvieron una mejor evaluación de sus funciones, situación que para el caso chileno podría entenderse ya que la generación de ciencia y tecnología históricamente ha estado desplegada por instituciones de educación superior. Este aspecto considerarse como un aprendizaje para la experiencia de observatorios, en el sentido de considerar la trayectoria institucional de las universidades para planificar procesos de investigación y generación de datos de investigación e información científica.

La experiencia de los Observatorios de turismo en Chile presentó brechas tanto en la consideración del turismo como una disciplina de estudio, como en la gestión de información y acceso a datos abiertos. En futuras instancias, un aspecto a considerar que puede extraerse del presente estudio, es la consideración fundacional de los aspectos ligados a datos de investigación e información científica, tanto en las características de la planificación, captura, almacenamiento, procesamiento, validación y diseminación. Ello permitiría incluso generar protocolos y coordinar los distintos observatorios. La diferencia de objetivos y mecanismos de cada uno impidió tal coordinación.

Finalmente, a pesar de lo compleja de la experiencia de los observatorios de turismo en Chile, dicha complejidad puede transformarse en una oportunidad para promover la actividad colaborativa entre universidades e instituciones públicas y mejorar los procedimientos con el que son obtenidos y difundidos los datos a través de los diversos actores que participan del sector. Con ello será posible continuar enriqueciendo el conocimiento sobre el turismo tanto para fines estrictamente disciplinares como vinculados a intereses productivos.

\section{AGRADECIMIENTOS}

Los autores agradecen el financiamiento de esta investigación a través del fondo DIUFRO DI15-0007 de la Universidad de La Frontera, a Patricia Muñoz, directora del Programa de Información Científica de la Comisión Nacional de Investigación Científica y Tecnológica y a los comentarios y sugerencias de la evaluación anónima de la Revista Cuadernos de Turismo.

\section{BIBLIOGRAFÍA}

ABADAL, E., OLLÉ CASTELLÀ, C., ABAD-GARCÍA, F. y MELERO, R. (2013): «Políticas de acceso abierto a la ciencia en las universidades españolas», Revista Española de Documentación Científica, vol. 36 (2), pp. 1-15.

AGENCIA AYSÉN. (2009): Diseño de Modelo de Observatorio Turístico Aysén» Agencia Regional Desarrollo Productivo. Marzo de 2009. 
BIZ, A, MICHELOTTI BETTONI, E y SOUZA NUNES, D. (2011): «Gestión participativa del turismo. Un análisis sobre el uso de las herramientas de las TIC'S por parte de los organismos públicos de turismo», Estudios y Perspectivas en Turismo, vol. 20 (2), pp. 327-340.

BURNEO, P y LÓPEZ, S. (2013): “"Observatorio de turismo”. Un espacio de análisis y Comunicación como contribución para el desarrollo del Turismo en el Ecuador», Gestión Turística, vol. 1 (19), pp. 9-24.

CAMPODÓNICO, R. y LUIS CHALAR BERTOLOTTI, L. (2011): «Hacia la construcción del conocimiento en turismo», Estudios y Perspectivas en Turismo, vol. 20 (6), pp. 1.307-1.323

CANCINO, R.; SEGUEL, A. (2014): «Condicionantes sociotécnicas de las decisiones políticas. El tsunami del 27F en Chile», Revista CTS, vol. 9 (251), pp. 161-180.

CONICYT; IDER. (2010): Estado del Arte Nacional e Internacional Sobre Manejo y Políticas de Acceso a Datos de Investigación e Información Científica y Tecnológica Financiada con Fondos Públicos y Recomendaciones de Buena Prácticas. Santiago de Chile, Gobierno de Chile.

CONICYT. (2014): Datos científicos abiertos. Santiago de Chile, Gobierno de Chile.

CORFO. (2015): Bienes Públicos para la Competitividad. Subdirección de Difusión Tecnológica y Entorno para la Innovación. Santiago de Chile, Gobierno de Chile.

CORFO; INE; SERNATUR; Universidad Arturo Prat. (2013): Estudio de Demanda Turística Región de Tarapacá. Gobierno Regional, Observatorio Turístico Región de Tarapacá.

DE FILIPPO, D., SANZ-CASADO, E., URBANO SALIDO, C., ARDANUY, J. y GÓMEZ-CARIDAD, I. (2011): «El papel de las bases de datos institucionales en el análisis de la actividad científica de las universidades», Revista Española de Documentación Científica, vol. 34 (2), pp. 165-189.

FNDR. Fondo Nacional de Desarrollo Regional. http:/www.subdere.gov.cl/programas/ divisi\%C3\%B3n-desarrollo-regional/fondo-nacional-de-desarrollo-regional-fndr [28 de junio de 2016]

GARCÍA HERNÁNDEZ, M. (2007): «Entidades de planificación y gestión turística a escala local. El caso de las Ciudades Patrimonio de la Humanidad de España», Cuadernos de Turismo, n ${ }^{\circ}$ 20, pp. 79-102.

GOBIERNO REGIONAL BIOBÍO. (2015): Política Regional de Turismo Región del Biobío. Gobierno Regional, Informe Etapa 4.

GONZÁLEZ, Z., CASTRO, M. y MARÍN, A. (2013): «Determinación de necesidades de investigación en turismo: El Caso del Centro de Investigación en Turismo y Patrimonio de la Región de Valparaíso, Chile». Estudios y Perspectivas en Turismo, vol. 22 (5), pp. 926-952.

JAFARI, J. (2005): «El turismo como disciplina científica. Política y Sociedad», vol. 42 (1), pp. 39-56.

MARTINI MOESCH, M. (2013): «El origen del conocimiento. El lugar de la experiencia y de la razón en la génesis del conocimiento del turismo», Estudios y Perspectivas en Turismo, vol. 22 (5), pp. 985-1001. 
MMA. Ministerio del Medio Ambiente.(2014): Estrategia Nacional de Turismo 20122020. Santiago de Chile, Gobierno de Chile.

MELERO, R. y HERNÁNDEZ-SAN-MIGUEL, J. (2014): «Acceso abierto a los datos de investigación, una vía hacia la colaboración científica», Revista Española de Documentación Científica, vol. 37 (4), pp.1-11.

MENDO, C, RAMOS, L.F., ARQUERO, R., DEL VALLE-GASTAMINZA, F., BOTEZÁN, I., SÁNCHEZ, R., TEJADA, C., PEÓN, J. L., COBO, S. y SALA, A. (2013): «Del acceso a la reutilización, del dato al documento: una visión conceptual de la información pública», Revista Española de Documentación Científica, vol. 36 (3), pp. 1-12.

PADILLA NAVARRO, P.A., CURAQUEO PICHIHUECHE, O.J., CANCINO SALAS, R.D. y GATICA ÁLVAREZ, M.H. (2013): «Acceso a datos de investigación e información científica en Chile», Revista Española de Documentación Científica, vol. 36 (3), pp. 1-7.

PICAZO PERAL, P. y MORENO GIL, S. (2012): «Difusión de la investigación científica de turismo en Chile y Argentina», Gestión Turística, nº 18, pp. 9-49.

PICAZO PERAL, P. y MORENO GIL, S. (2013): «Difusión de la investigación científica en turismo. El caso de México», El Periplo Sustentable, n 24, pp. 7-40.

PILAT, D. y FUKASAKU, Y. (2007): «OECD Principles and Guidelines for Access to Research Data from Public Funding», Data Science Journal, vol. 17, pp. 4-11.

SANHUEZA, P., RODRÍGUEZ, I. y PADILLA, P. (2013): «Difusión y Acceso a Datos e Información Científica y Tecnológica en la Normativa de Fondos Públicos para la Innovación en Chile», Journal of Technology Management \& Innovation, vol. 7 (4), pp. 148-163.

SANTÁGATA, H. (2011): «La Importancia de un Observatorio Turístico en la Provincia de Buenos Aires como Instrumento de orientación en la toma de decisiones y en la planificación», Notas en Turismo y Economía, no 3, pp. 8-44.

SAMPIERI, R., FERNÁNDEZ, C. y BAPTISTA, P. (2010): Metodología de la investigación ( $5^{\mathrm{a}}$ ed.). México, McGraw Hill.

OBSERVATORIO TURÍSTICO DEL MAULE. (2012): Memoria Anual y Recopilación de boletines Informativos 2011-2012. Facultad de Ciencias Empresariales, Universidad de Talca.

VELARDE-VALDEZ, M., SANTILLÁN-NÚÑEZ, M.A. y OBOMBO-MAGIO, K. (2015): «Tecnologías de Información y Comunicación al servicio del turismo en Mazatlán, Sinaloa, México. Ciencias Holguín, vol. 21, pp. 1-10. 